\title{
Shigella Enterotoxin 1: An Enterotoxin of Shigella flexneri 2a Active in Rabbit Small Intestine In Vivo and In Vitro
}

\author{
Alessio Fasano, ${ }^{\star * \|}$ Fernando R. Noriega, ${ }^{*}$ David R. Maneval, Jr., ${ }^{\star \|}$ Somchoke Chanasongcram, ${ }^{\star}$ Robert Russell, *\|! \\ Stefano Guandalini, ** and Myron M. Levine, ${ }^{\star \$ \|}$ \\ *The Center for Vaccine Development, Divisions of ${ }^{\ddagger}$ Pediatric Gastroenterology and Nutrition and ${ }^{\S}$ Infectious Diseases and Tropical \\ Pediatrics, Department of Pediatrics; "Division of Geographic Medicine, Department of Medicine; 'Department of Pathology, University \\ of Maryland School of Medicine, Baltimore, Maryland 21201; and **the Pediatric Unit, University of Catanzaro School of Medicine, \\ 88100 Catanzaro, Italy
}

\begin{abstract}
Culture filtrates of Shigella flexneri 2a strain M4243 grown in iron-depleted medium, caused significant fluid accumulation in rabbit ileal loops. Also, when tested in Ussing chambers, a greater rise in potential difference and short circuit current was seen with such filtrates compared with the medium control. Analogous filtrates from two M4243 derivatives lacking the 140-MD invasiveness plasmid (either M4243avir or BS103) retained 60-65\% of the wild-type enterotoxic activity. Ultrafiltration and gel exclusion size fractionation of M4243 filtrate revealed that the activity was $\sim 60 \mathrm{kD}$. SDS-PAGE performed on this fraction showed 18 bands, 5 of which reacted with human convalescent sera. Genes encoding this enterotoxin, named ShET1 for Shigella enterotoxin 1 , were cloned from the $S$. flexneri 2 a chromosome, and two separate open reading frames of 534 and 186 bp were sequenced. These observations suggest that $S$. flexneri 2a elaborates two distinct enterotoxins: ShET1, encoded by genes located on the chromosome, and ShET2, encoded by a gene on the 140-MD invasiveness plasmid. ShET1, which is composed of two distinct subunits and is elaborated in vivo, where it elicits an immune response, may be important in the pathogenesis of diarrheal illness due to S. flexneri 2a. (J. Clin. Invest. 1995. 95:2853-2861.) Key words: diarrhea - immune response - invasion • cytotoxin • Ussing chamber
\end{abstract}

\section{Introduction}

Although the cardinal feature in the pathogenesis of Shigella flexneri infection involves the invasion of epithelial cells, it nevertheless has been hypothesized that $S$. flexneri also produces an enterotoxin $(1,2)$. Support for this hypothesis was obtained by Rout et al. (1), who documented jejunal secretion in experimental shigellosis in monkeys challenged with the prototype wild-type $S$. flexneri 2a strain M4243. We have previously re-

Address correspondence to Dr. Alessio Fasano, Center for Vaccine Development, University of Maryland School of Medicine, 10 South Pine Street, Baltimore, MD 21201. Phone: 410-706-5328; FAX: 410-7066205; E-mail: afasano@umabnet.ab.umd.edu.

Received for publication 3 November 1994 and in revised form 10 February 1995.

J. Clin. Invest.

(c) The American Society for Clinical Investigation, Inc.

0021-9738/95/06/2853/09 \$2.00

Volume 95, June 1995, 2853-2861 ported that, when grown in iron-depleted medium, enteroinvasive Escherichia coli (EIEC) ${ }^{1}$ elaborate an enterotoxin (EIET; 68-80 kD) that causes fluid accumulation in isolated rabbit ileal loops and an electrical response in Ussing chambers (3). Based on the similarities known to exist between EIEC and Shigella (4), we investigated the possibility that $S$. flexneri 2 a expresses an enterotoxin(s). Our group has recently cloned and sequenced the EIET gene (5). We then found virtually the identical gene (99\% homology) on the 140-MD invasiveness plasmid of S. flexneri 2a (5), which we refer to as Shigella enterotoxin 2 (ShET2). In this report, we describe a second enterotoxin elaborated by $S$. flexneri $2 \mathrm{a}$, its neutralization by homologous antitoxin, the distinction between the observed enterotoxicity and cytotoxicity, and the cloning and DNA sequencing of the chromosomal genes encoding the toxin.

\section{Methods}

\section{Bacterial strains}

The strains used in the present paper are listed in Table I. S. flexneri 2a strain M4243 was used for our animal studies, since Rout et al. (1) showed that, when fed to monkeys, this strain induced secretory diarrhea. M4243 and its plasmid-cured derivative M4243avir were obtained from the Walter Reed Army Institute of Research, Washington, D.C. M4243avir is a spontaneous plasmid-cured derivative of M4243 obtained after several passages of the wild-type strain at room temperature. To rule out the possibility that spontaneous mutations may have occurred in the genomic chromosome of M4243 that could account for the decreased enterotoxic activity of M4243avir, a second plasmid-cured derivative, BS103 (6), was tested. BS103, kindly provided by Dr. Anthony Maurelli of the Uniformed Services University of the Health Sciences, Bethesda, MD, was obtained from the wild-type strain after a limited number of passages. S. flexneri 2a strain 2457T, which has been routinely used in volunteer challenge studies since the late 1960s (7), was cured of the 140-MD invasiveness plasmid (2457TA) and used to clone the gene encoding the enterotoxic moiety. It should be noted that strain M4243 represents a clone isolated from the diarrheal stools of a monkey fed $2457 \mathrm{~T}$ and that it is indistinguishable from 2457T. S. flexneri $2 \mathrm{a}$ strains $2457 \mathrm{~T}$ and its plasmid-cured derivative 2457TA (low passage) came from the Center for Vaccine Development strain collection. Enterohemorrhagic $E$. coli (EHEC) strain 933 (serotype O157:H7), which elaborates Shiga-like toxin I and II, was used as the positive control in the Vero cell cytotoxicity assay (3). Nonpathogenic $E$. coli strain HS has been used extensively as a negative control in assays of pathogenicity and in clinical studies $(8,9)$. The bacterial isolates were initially cultured on blood agar to assess their purity. Isolated colonies were

1. Abbreviations used in this paper: EHEC, enterohemorrhagic Escherichia coli; EIEC, enteroinvasive $E$. coli; $I_{\mathrm{sc}}$, short circuit current. MW, molecular weight; orf, open reading frame; ShET, Shigella enterotoxin. 
Table I. Description of the Strains Used in the Current Study

\begin{tabular}{|c|c|c|c|c|c|}
\hline Strain & Sereny Test & Diarrhea in monkeys & Diarrhea in humans & Comments & Reference \\
\hline S. flexneri $2457 \mathrm{~T}$ & + & + & + & S. flexneri $2 \mathrm{a}$ wild type & 7 \\
\hline S. flexneri $2457 \mathrm{TA}$ & - & ND & ND & Plasmid-cured derivative of $2457 \mathrm{~T}$ & This paper \\
\hline S. flexneri M4243 & + & + & ND & Isolate from stool of monkey that ingested $2457 \mathrm{~T}$ & 12 \\
\hline S. flexneri M4243avir & - & - & ND & High passage derivative of M4243 & 22 \\
\hline S. flexneri BS103 & - & - & ND & Low passage derivative of M4243 & 6 \\
\hline E. coli $\mathrm{HS}$ & - & - & - & Nonpathogenic $E$. coli & 8,9 \\
\hline
\end{tabular}

subcultured to casamino acids-yeast extract agar and incubated in ambient air overnight at $37^{\circ} \mathrm{C}$

\section{Culture conditions and preparation of culture filtrate fractions}

All the aforementioned $S$. flexneri 2a strains were grown for $48 \mathrm{~h}$ at $37^{\circ} \mathrm{C}$ with shaking ( $200 \mathrm{rpm}$ ) in Chelex ${ }^{\circledR}$-treated (Bio Rad Laboratories, Richmond, CA), iron-depleted syncase broth (10) in iron-free culture vessels. Supernatants were collected by centrifugation at $12,000 \mathrm{~g}$ for $20 \mathrm{~min}$ at $4^{\circ} \mathrm{C}$ and passed through a $0.22-\mu \mathrm{m}$ membrane filter.

Culture filtrates of M4243avir were size fractionated using a series of Diaflo ultrafilters (Amicon Corp., Danvers, MA) to approximate the molecular weight (MW) of the material eliciting enterotoxic activity in rabbit ileal loops and in Ussing chambers. YM100 (100,000 MW cutoff), YM30 (30,000 MW cutoff), and YC05 (500 MW cutoff) membranes were used sequentially to fractionate the culture supernatants. Each membrane retentate was washed free of lower molecular weight species and exchanged into PBS, $\mathrm{pH} 7.3$, on each membrane by two successive 1:10 dilutions with PBS, followed by concentration and final reconstitution to the original supernatant volume in PBS. Resulting supernatant fractions representing coarse pools of $>100,30-100$, and $0.5-30 \mathrm{kD}$ were assayed for activity in rabbit ileal loops, Ussing chambers, and Vero cells.

\section{Partial purification of enterotoxin}

The M4243avir material exhibiting enterotoxic activity (30-100 kD) was further fractionated by size exclusion chromatography using a column with a fractionation range of $10-80 \mathrm{kD}$ (Protein Pak 125; 7.8 $\times 300 \mathrm{~mm}$ with guard column; Millipore Corp., Waters Chromatography, Bedford, MA). The $30-100-\mathrm{kD}$ fraction was prepared as previously described and further concentrated 100 -fold on a YM30 membrane, and $200 \mu \mathrm{l}$ was applied to and eluted from the column with PBS at $0.5 \mathrm{ml} / \mathrm{min}$. The eluate absorbance at $280 \mathrm{~nm}$ was monitored, 0.5 $\mathrm{ml}$ fractions were collected, and those exhibiting absorbance were assayed for activity in rabbit ileal loops and Ussing chambers.

\section{Preparation of antitoxin in rabbits}

New Zealand White male rabbits were used to produce antibodies for neutralization experiments. After obtaining a preimmunization serum specimen, $1 \mathrm{ml}$ of the concentrated 30-100-kD enterotoxic fraction of M4243avir supernatants previously described was mixed with an equal volume of Freund's complete adjuvant and inoculated subcutaneously in four separate sites. A booster dose of pooled chromatographic fractions of $\sim 60 \mathrm{kD}$ exhibiting activity was administered with Freund's incomplete adjuvant $4 \mathrm{wk}$ later; 1 mo thereafter, the rabbit was bled.

For those experiments designed to identify ShET1 as expressed in an $E$. coli host, this antiserum was absorbed for $20 \mathrm{~min}$, three times, either with freshly harvested Epicurian coli strain XL1-Blue MRF' (pBluescript SK \pm ) (Stratagene, La Jolla, CA) or with E. coli K12 strain DH5 $\alpha$ (pBluescript SK \pm ) before its use in immunoblots.

\section{Human antitoxin}

Pre- and postchallenge pooled sera from 10 adult volunteers who developed diarrhea after ingesting S. flexneri 2a M4243 while serving as unimmunized controls in vaccine efficacy studies (11) were prepared for use in neutralization experiments in Ussing chambers and in immunoblotting procedures.

\section{Rabbit ileal loop test}

Adult New Zealand White rabbits were used to perform the experiments as previously described (3). Whole cultures of $S$. flexneri strain M4243 and its plasmid-cured derivative M4243avir, along with their respective sterile supernatants, were tested. Supernatant from nonpathogenic $E$. coli strain HS was also included in each experiment as a negative control (3). At the end of the experiments, sections of intestine from each loop were fixed in $10 \%$ formalinized saline to be examined by light microscopy.

\section{Ussing chambers}

The experiments were performed by methods previously described in extensive details (12). Eight pieces of rabbit small intestine were mounted in Ussing chambers, kept at $37^{\circ} \mathrm{C}$, and gassed with $95 \% \mathrm{O}_{2} /$ $5 \% \mathrm{CO}_{2}$. Once the tissue reached a steady-state condition, $300 \mu \mathrm{l}$ of filtered supernatants obtained from the strains to be tested was added to the mucosal surface, resulting in a 1:33 dilution of the original sample ( $0.3 \mathrm{ml}$ into $10 \mathrm{ml}$ of bathing solution). An identical sample was also added to the serosal side in each chamber to preserve osmotic balance. Transepithelial electrical potential difference was measured at intervals, and total tissue conductance and short circuit current $\left(I_{\mathrm{sc}}\right)$ were calculated (12). A known positive and appropriate negative control including culture medium or HS supernatant were always assayed in parallel with the test samples using the same rabbit tissue.

\section{Neutralization of enterotoxic activity}

Experiments to neutralize enterotoxic activity were performed in Ussing chambers. $600-\mu \mathrm{l}$ aliquots of active $30-100-\mathrm{kD}$ fraction of either M4243avir or clones containing set 1 gene supernatants were mixed and incubated for $60 \mathrm{~min}$ with $60 \mu \mathrm{l}$ of the pre- and postimmune rabbit sera or the pre- and post $-S$. flexneri challenge pooled sera from human volunteers.

\section{Cytotoxicity assay}

Cell lysates were obtained from cells harvested from cultures of strain M4243avir by centrifugation at $12,000 \mathrm{~g}$ for $20 \mathrm{~min}$ at $4^{\circ} \mathrm{C}$. Cytotoxicity assays were performed on Vero cells as previously described (3) by using the method of Gentry and Dalrymple (13). Cytotoxic titers were calculated as the reciprocal of the $50 \%$ cytotoxic dose per $\mathrm{mg}$ of protein in culture supernatant or cell lysate; the protein content was measured by the method of Bradford (14).

\section{Large-scale production and purification of Shigella enterotoxin 1}

Large scale preparation of $S$. flexneri 2a enterotoxin 1 was undertaken in order to obtain sufficient material for further characterization and analysis. Plasmid-minus $S$. flexneri 2 a was inoculated into 30 liters of $L$ broth containing the iron chelator ethylenediamine-di-o-hydroxyphenyl acetic acid (15) at $25 \mu \mathrm{g} / \mathrm{ml}$ and incubated overnight at $37^{\circ} \mathrm{C}$ in a 30 liter fermentor (New Brunswick Scientific, Edison, NJ). Bacterial cells 
were removed by centrifugation, and the supernatant was filtered. This filtrate was fractionated and concentrated 100-fold within the 30-100$\mathrm{kD}$ range as previously described except a Pellicon tangential flow ultrafiltration apparatus and membrane cassettes (Millipore Corp., Waters Chromatography) were used. A $10-\mathrm{ml}$ aliquot of the $30-100-\mathrm{kD}$ concentrate was then further fractionated by replicate separations with an HPLC size exclusion column (SEC-2000; $7.5 \times 600 \mathrm{~cm}$ with guard column; Phenomenex, Torrance, CA) as previously described. The fractions containing moieties in the $60-\mathrm{kD}$ range were collected, pooled, and concentrated by vacuum dialysis to $1 \mathrm{ml}$ using a 10-kD membrane (MicroProDiCon; Spectrum Medical Industries, Los Angeles, CA). This material was assayed for enterotoxic activity and separated by SDSPAGE (16) using a preparative well. Resultant bands were transferred to a nitrocellulose membrane by the method of Towbin et al. (17). Multiple vertical strips of the nitrocellulose membrane were prepared and stained with colloidal gold (Aurodye; Janssen Pharmaceutica, Piscataway, NJ) to visualize protein bands, or reacted with the pooled convalescent sera from volunteers challenged with wild-type $S$. flexneri 2 a by previously described Western immunoblotting techniques (18).

\section{Cloning of the genes encoding ShETI}

Library construction. To clone and characterize the chromosomal gene(s) encoding ShET1, a genomic library of strain 2457TA was prepared. Genomic DNA of 2457TA was partially digested with Sau3A, the 5-10-kb fragments were purified by GeneClean (BIO 101, La Jolla, CA), and the Sau3A DNA termini were partially filled with DATP and dGTP in a Klenow reaction. In parallel, the cos ends of undigested $\lambda Z A P I I$ vector (Stratagene) were ligated, the vector was digested with XhoI, and the resulting termini were partially filled with dCTP and dTTP. This resulted in compatible ends between the vector and genomic inserts, but not between themselves, that were ligated and packaged with Gigapack II Gold packaging extract (Stratagene) system following the procedures recommended by the manufacturer. This XZAPII::2457TA library was titrated in E. coli strain XL1-Blue MRF' to obtain a concentration of 100 plaques per $100-\mathrm{mm}$ plate.

Detection of library clones expressing ShETI. Plaques were blotted with isopropyl-thio- $\beta$-D-galactopyranoside-saturated nitrocellulose filters following standard procedures for immunological screening of expression of bacteriophage $\lambda$ vector libraries (19). The positive plaques were harvested, and the phagemids (pBluescript SK \pm ) containing the corresponding 2457TA DNA inserts were excised from the $\lambda$ ZAPII vector using the ExAssist/SOLR (Stratagene) system following the manufacturer's recommendations. 24 single colonies derived from each immunoblot-positive plaque were grown in $300 \mu$ of iron-depleted LB medium with ampicillin in 96-well microtiter plates and cultured at $37^{\circ} \mathrm{C}$ for $48 \mathrm{~h}$. The supernatants of these cultures were freeze-thawed three times, passed by gravity through nitrocellulose paper in a 96well manifold (Bio Rad Laboratories), and immunoblotted with the previously described rabbit antiserum. Selected phagemids were purified and electroporated in E. coli K12 strain DH5 . Supernatants derived from these clones, along with their controls, were precipitated with TCA, concentrated $30 \times$, subjected to SDS-PAGE on nitrocellulose papers, and immunoblotted with the aforementioned absorbed rabbit antiserum.

Sequencing. Single-strand sequencing of the selected genomic insert in pBluescript $\mathrm{SK} \pm$ (pF9-1-90) was performed by automated fluorescence sequencing (DNA sequencer model 373A; Applied Biosystems, Inc., Foster City, CA). The cDNA strand was sequenced by chain termination sequencing using the Sequenase Version 2.0 DNA sequencing kit (United States Biochemical Corp., Cleveland, $\mathrm{OH}$ ). Chain termination sequencing was used as well to identify and determine the orientation of the set 1 genes in pset 1 (see the following section).

Cloning of set 1 genes. The 1,093-bp fragment containing the set 1 open reading frames (orfs; with an upstream segment of $98 \mathrm{bp}$ ) was obtained by digesting the 6-kb insert in pF9-1-90 with XmaI and cloning it in pBluescript SK \pm . The plasmid so obtained, named pset 1 , was transformed in DH5 $\alpha$. DH5 $\alpha$ (pset 1) crude supernatants were then immunoblotted as previously described and tested in Ussing chambers for enterotoxic activity. The band of $\sim 10 \mathrm{~cm}$ in length corresponding to
Table II. Rabbit Ileal Loop and Ussing Chamber Experiments

\begin{tabular}{lcc}
\hline \multicolumn{1}{c}{ Sample $(n)$} & $\begin{array}{c}\text { Ileal loops } \\
\text { (fluid accumulatiion) }\end{array}$ & $\begin{array}{c}\text { Ussing chambers } \\
\text { (delta } I_{\text {sc }} \text { ) }\end{array}$ \\
\hline & $m l / c m$ & $\mu A m p / \mathrm{cm}^{2}$ \\
M4243 bacteria (5) & $1.06(0.34)^{*}$ & $\mathrm{ND}$ \\
M4243 supernatant (5) & $0.53(0.09)^{*}$ & $29.5(5.1)^{\ddagger}$ \\
M4243avir bacteria (8) & $0.19(0.06)^{8}$ & $\mathrm{ND}$ \\
M4243avir supernatant (9) & $0.21(0.03)^{\xi}$ & $19.3(2.9)^{\xi}$ \\
BS103 supernatant (5) & $\mathrm{ND}$ & $17.7(3.5)^{\xi}$ \\
HS supernatant (11) & $0.05(0.01)$ & $4.1(2.2)$ \\
& &
\end{tabular}

Results are expressed as means (SE) for the number of animals given in parentheses. ND, not done. ${ }^{*} P<0.0005,{ }^{\dagger} P<0.01,{ }^{8} P<0.05$, compared with HS negative control.

the 55-kD immunoreactive material was carefully excised with a scalpel. Material from this band was eluted by dissolution of the nitrocellulose in $200 \mu \mathrm{l}$ of DMSO, addition of $4 \mathrm{vol}$ of water to precipitate the nitrocellulose, centrifugation, and dialysis of the supernatant. The sample was then tested for enterotoxic activity in Ussing chambers.

\section{Statistics}

All results are expressed as means \pm SE. The significance of the differences was calculated using Student's $t$ test for either paired or unpaired variates.

\section{Results}

\section{Rabbit ileal loop test}

The intestinal loops injected with whole viable cultures of M4243 showed pronounced fluid accumulation at $18 \mathrm{~h}$ after inoculation (Table II). On histologic examination, severe tissue damage was observed, characterized by prominent necrosis of the luminal epithelium and marked villus atrophy (Fig. $1 a$ ). It is notable that although M4243 sterile culture supernatant also induced a significant fluid accumulation as compared with the HS negative control (Table II), no tissue damage was detected (Fig. $1 b$ ). Fluid accumulation induced by M4243avir (both whole culture and sterile supernatant) was lower than M4243 supernatant-induced secretion, but still significantly higher than the negative control strain HS (Table II); no tissue damage was observed in the positive loops (data not shown). The results of experiments clearly show that iron-limited culture conditions are required in order to detect expression of M4243 enterotoxic activity in sterile supernatants (Table III). Expression was not notably affected by the length of incubation (Table III).

\section{Ussing chambers}

The results obtained in the rabbit ileal loop assays were compatible with elaboration of an enterotoxin by M4243. To test this hypothesis, sterile crude supernatants obtained from iron-limited M4243, M4243avir, and BS103 cultures were tested in rabbit small intestine mounted in Ussing chambers. A 30-100$\mathrm{kD}$ supernatant fraction from EIEC CVD/EI-34 $(\mathrm{O} 136: \mathrm{H}-)$ and HS supernatant were used as positive and negative controls, respectively. As shown in Table II, the overall increase in $I_{\text {sc }}$ was significantly greater for M4243 supernatant as compared with the HS supernatant negative control. Repeated assays showed that supernatants from both of the plasmid-minus 

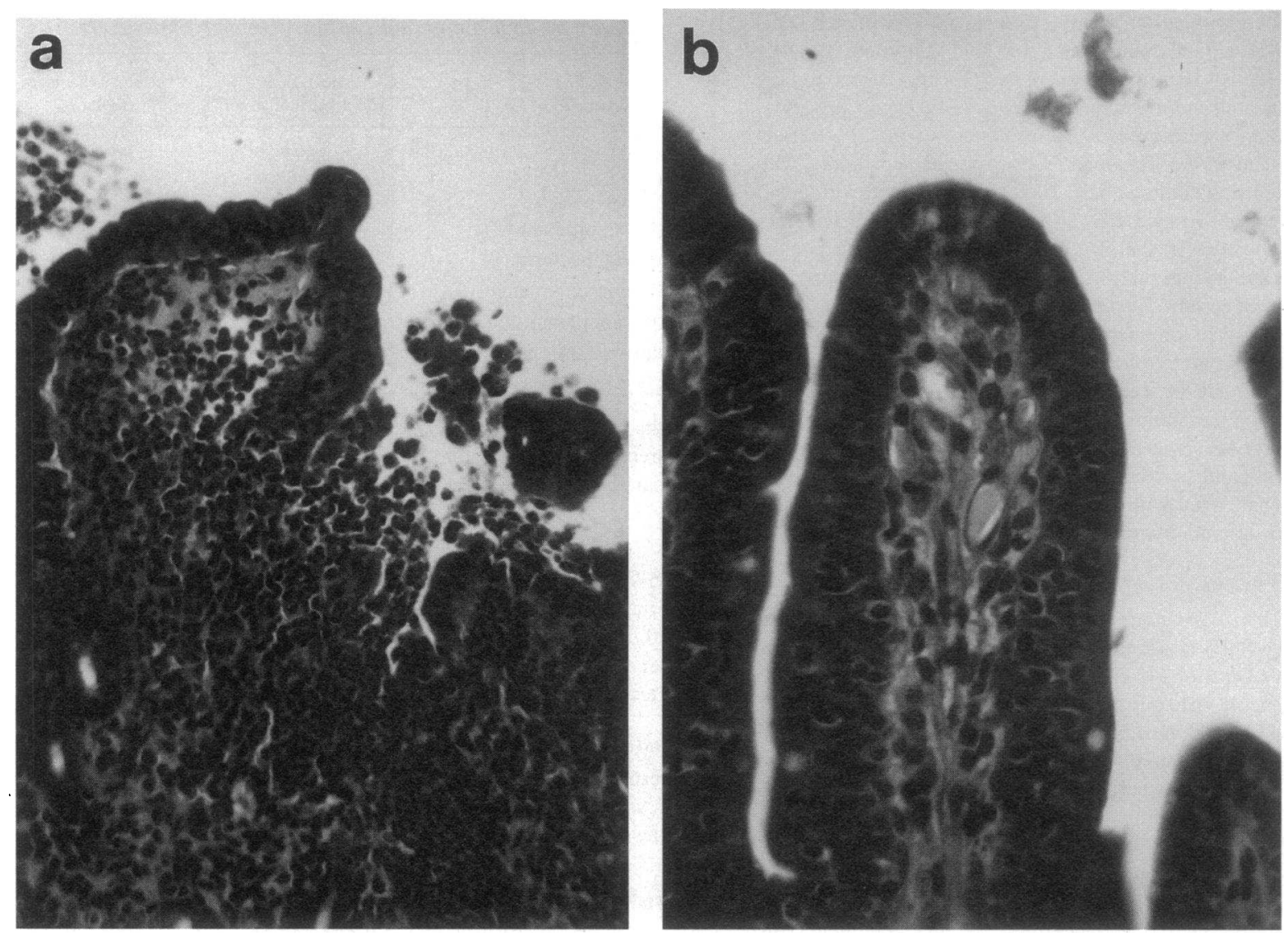

Figure 1. Light micrographs of rabbit ileal mucosa exposed for $18 \mathrm{~h}$ to S. flexneri 2a M4243 whole culture and sterile supernatant. (a) Light microscopy of damage to rabbit villus epithelium challenged with S. flexneri 2a M4243 whole culture. There is extensive necrosis of the epithelial cells on the surface of the villi resulting in villus atrophy. (b) Loop of intestine inoculated with $S$. flexneri 2a M4243 sterile supernatant. Normal histology of rabbit ileal mucosa. Light microscopy examination was conducted $18 \mathrm{~h}$ after inoculation. Hematoxylin and eosin stain. Magnification, 40 .

strains, M4243avir and BS103, expressed significantly less enterotoxin in comparison with the plasmid-containing M4243 (Table II; $P<0.05$ ). However, the enterotoxic activity of the M4243avir and BS103 supernatants was nevertheless significantly greater than the negative control (Table II). These data suggest that, though not absolutely necessary for the effect, the virulence plasmid of $S$. flexneri confers enhanced enterotoxic

\section{Table III. Fluid Accumulation in Rabbit Ileal Loops}

\begin{tabular}{ll}
\hline Growth conditions & Fluid accumulation \\
\hline & $m / / c m$ \\
M4243 supernatants & \\
L broth, 24 h (4) & $0.01 \pm 0.01$ \\
L broth, 72 h (4) & $0.04 \pm 0.03$ \\
Minimal iron broth, 24 h (4) & $0.43 \pm 0.11^{*}$ \\
Minimal iron broth, 72 h (4) & $0.47 \pm 0.14^{*}$ \\
HS supernatant & \\
Minimal iron broth, 24 h (4) & $0.01 \pm 0.01$ \\
\hline
\end{tabular}

Results are expressed as mean $\pm \mathrm{SE}$ for $(n)$ animals. The minimal iron broth tested was Chelex-deferrated syncase. ${ }^{*} P<0.01$ compared with HS. activity. We have recently cloned and sequenced a 1,595-bp orf localized on the $S$. flexneri 2 a plasmid that encodes a distinct enterotoxin (ShET2). The ShET2 gene shares 99\% homology with the EIET gene of EIEC (5). We therefore turned the focus of further investigations to the chromosome-related enterotoxin (ShET1). The experiments described in the following text were performed by using plasmid-cured derivatives of $S$. flexneri $2 \mathrm{a}$.

\section{Neutralization}

The electrical response in Ussing chambers was completely abolished when M4243avir supernatant was preincubated either with polyclonal rabbit antibodies raised against an active $30-$ $100-\mathrm{kD}$ fraction of such supernatant (Fig. $2 a$ ) or with convalescent sera from volunteers who had been challenged with $S$. flexneri 2a (Fig. $2 b$ ). Neutralization was not observed with the preimmune sera, in which responses were similar to those seen when testing the active fraction alone.

\section{Cytotoxicity assay}

Both supernatant and lysate of the positive control EHEC O157:H7 strain 933 showed a high level of cytotoxicity (5.0 $\times 10^{3}$ and $3.4 \times 10^{4} \mathrm{CD}_{50}$ per $\mathrm{mg}$ of protein, respectively). In contrast, neither supernatant nor lysate from negative control strain HB101 showed cytotoxic activity. Against these two extremes, M4243avir exhibited low cytotoxic activity in the super- 

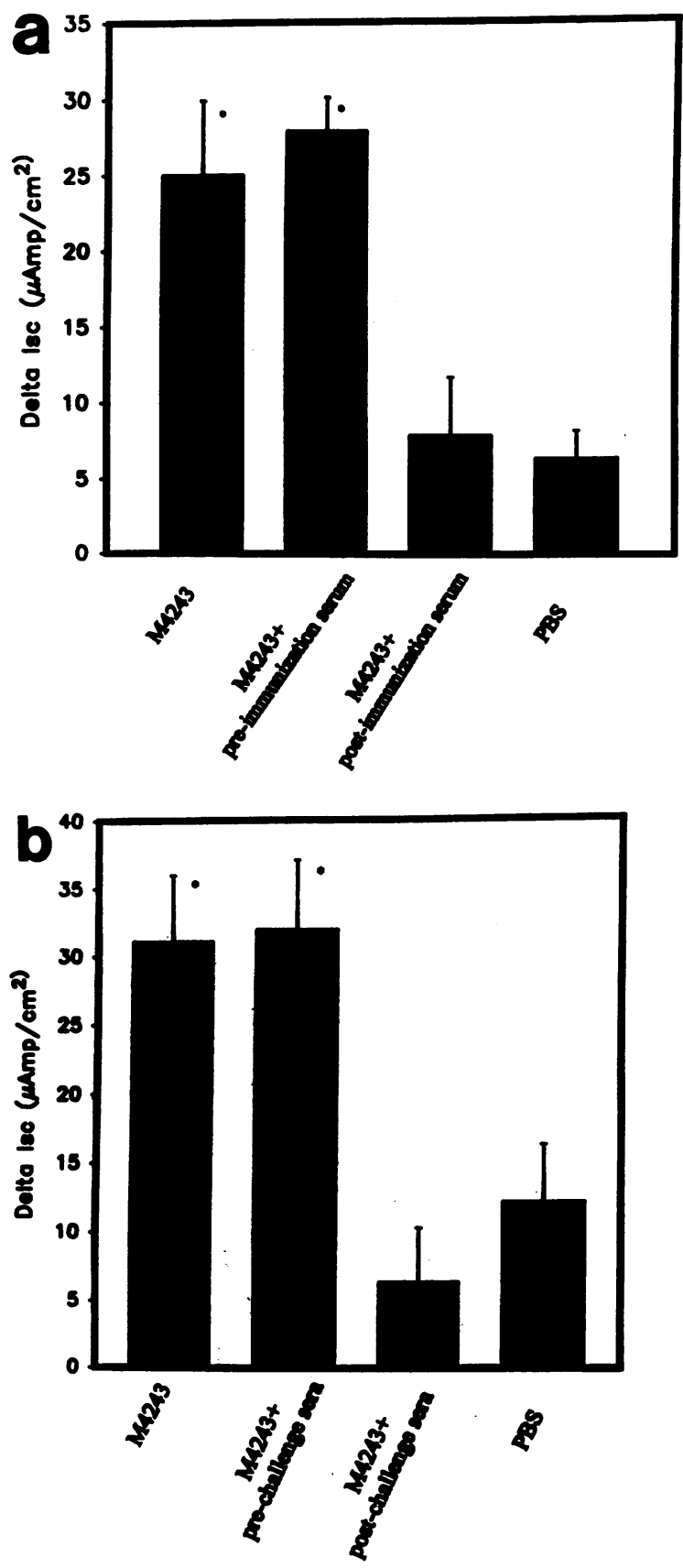

Figure 2. Neutralization experiments in Ussing chambers. (a) Peak increments induced by the 30-100-kD fraction from iron-limited M4243avir culture supernatants, alone or preincubated with either preimmune or immune serum produced in rabbits. Number of animals $=4$. Values are mean \pm SE. $* P<0.02$ compared with the negative control (PBS). (b) Peak increments induced by the 30-100-kD fraction from iron-limited M4243avir culture supernatants, alone or preincubated with serum obtained from volunteers before or after challenge with $S$. flexneri 2a. Number of animals $=4$. Values are mean \pm SE. $* P<0.02$ compared with the negative control (PBS).

natant $\left(4.2 \times 10^{2} \mathrm{CD}_{50}\right.$ per $\mathrm{mg}$ of protein) and lysate (5.1 $\times 10^{2} \mathrm{CD}_{50}$ per $\mathrm{mg}$ of protein).

\section{Preliminary estimate of enterotoxin size}

To obtain a preliminary estimate of the molecular mass of the enterotoxin and to establish the correlation of enterotoxic and
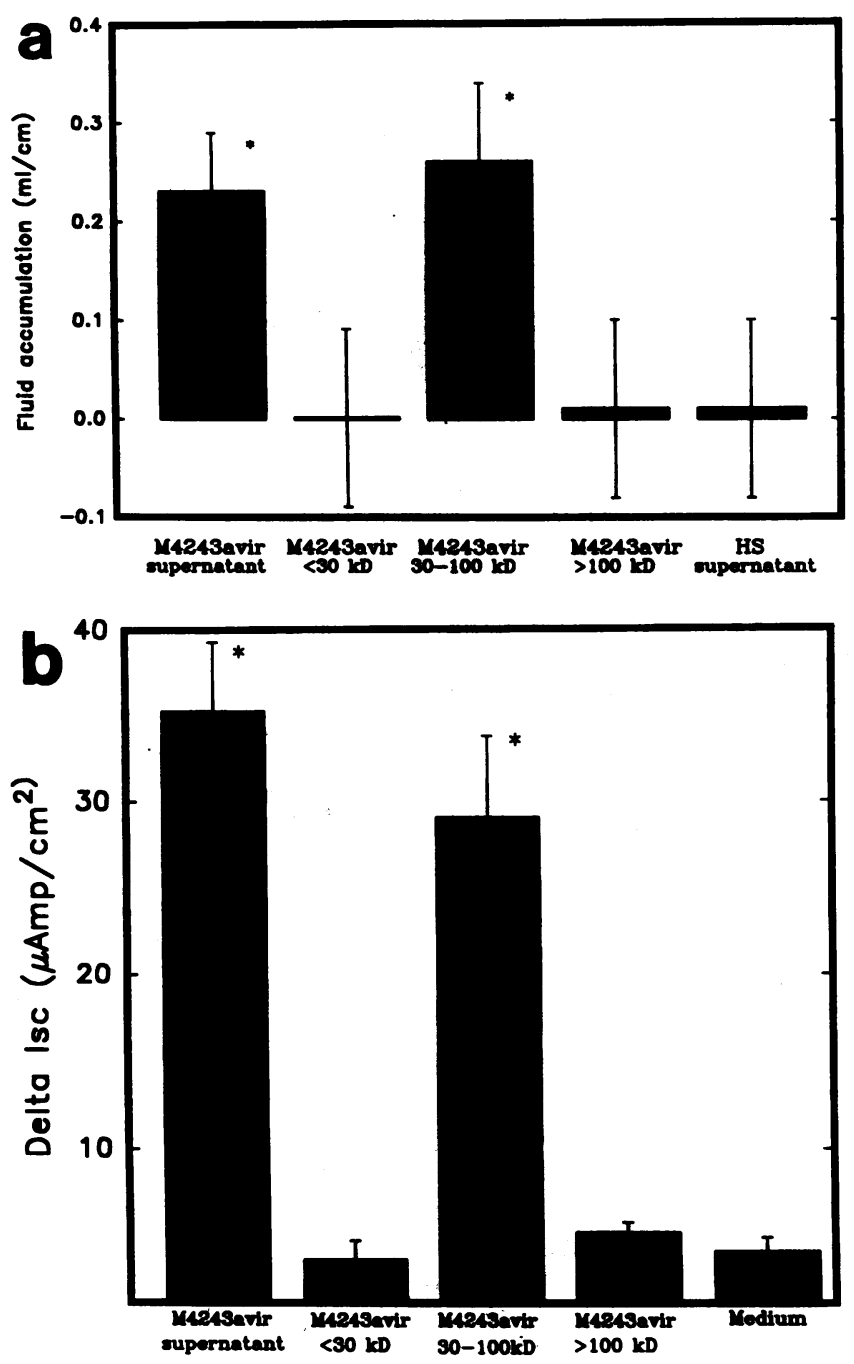

Figure 3. Estimation of molecular mass range of enterotoxin. Material from $S$. flexneri 2a strain M4243avir supernatant fractionated by ultrafiltration into molecular mass ranges and assayed for enterotoxic activity in rabbit ileal loops $(a)$ and Ussing chambers $(b)$. Nonpathogenic $E$. coli HS supernatant and Chelex-treated syncase medium were negative controls. Number of animals $=4$. Values are mean \pm SE. ${ }^{*} P<0.05$ compared with other fractions and HS supernatant $(a) .{ }^{*} P<0.02$ compared with other fractions and the medium control $(b)$.

cytotoxic activities, supernatants from M4243avir grown in iron-limited medium were fractionated by membrane ultrafiltration. Three coarse size fractions were tested for enterotoxic activity in Ussing chambers and ileal loops and for cytotoxicity in Vero cells. As shown in Fig. 3, both ileal loop (Fig. $3 a$ ) and Ussing chamber (Fig. $3 \mathrm{~b}$ ) assays localized the active enterotoxic fraction within the $30-100-\mathrm{kD}$ size range. In contrast, the cytotoxic activity was detected exclusively in the $<30-\mathrm{kD}$ fraction (data not shown).

\section{Partial purification of the enterotoxin}

The 30-100-kD fraction exhibiting enterotoxic activity was further fractionated by size using HPLC. Material eluting at times equivalent to $\sim 60 \mathrm{kD}$ relative to molecular mass calibration standards was active in both ileal loops and Ussing chambers (Fig. 4). This subfraction contained the bulk of the activity 


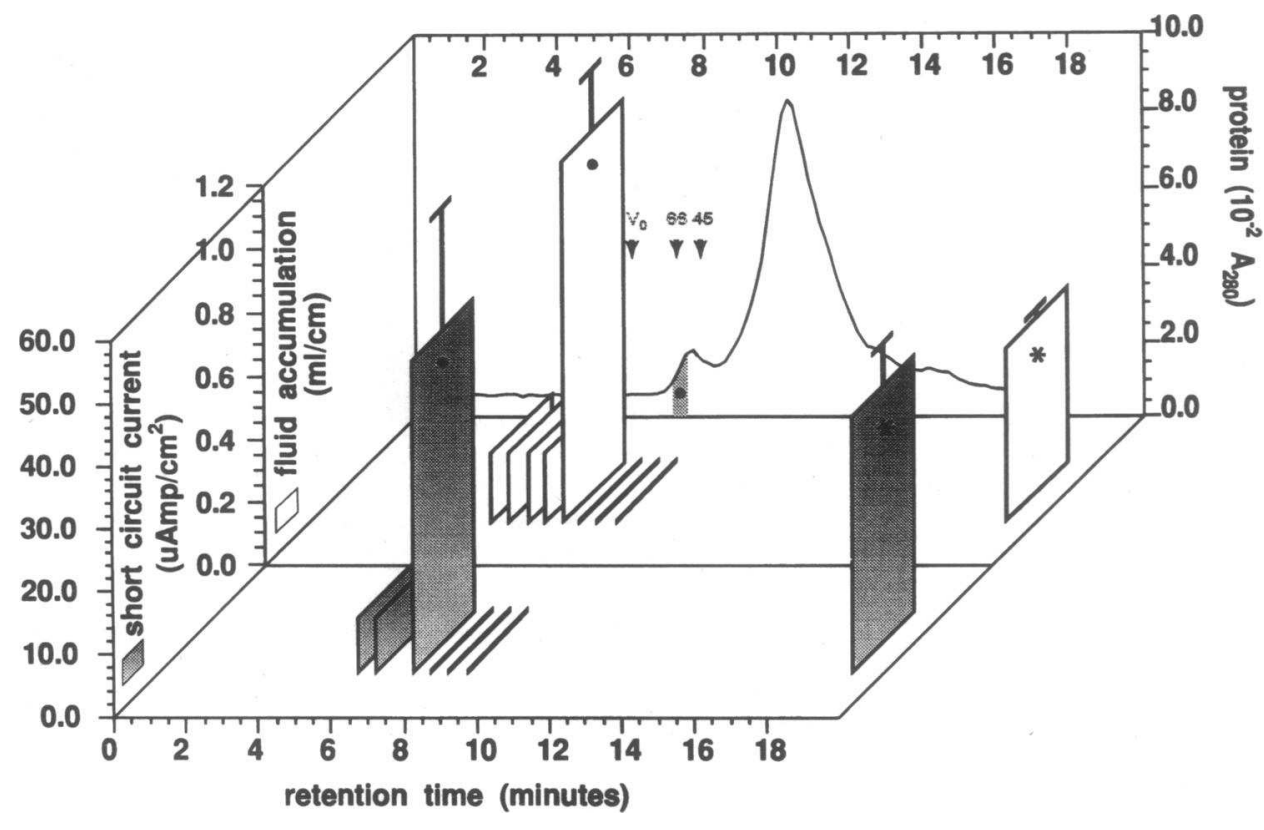

Figure 4. Enterotoxic activity of size exclusion chromatographic fractions in ileal loops and Ussing chambers. Material from filtered concentrated $30-100-\mathrm{kD}$ supernatants of M4243 grown in iron-depleted medium $(*)$ further fractionated by size exclusion chromatography and tested for activity in rabbit ileal loops and Ussing chambers. Open bars represent fluid accumulation ratios; shaded bars are $I_{\mathrm{sc}}$ variations. Values with error bars represent the averages of two separate experiments on two different preparations. Retention times for molecular mass standards $(\downarrow)$ and the $\sim 60-\mathrm{kD}$ fraction exhibiting the greatest activity $(\bullet)$ are indicated on the chromatographic tracing.

but only $5 \%$ of $280 \mathrm{~nm}$-absorbing material in the $30-100-\mathrm{kD}$ fraction.

\section{Further purification of enterotoxin}

Material from the large scale preparation representing a 100fold concentrate of $30-100-\mathrm{kD}$ supernatant from a 30-liter irondepleted culture of plasmid-cured $S$. flexneri 2 a exhibited enterotoxic activity similar to levels observed for smaller batches. Pooled size exclusion fractions of $\sim 60 \mathrm{kD}$ were shown to generate 18 bands when subjected to preparative SDS-PAGE, transferred to nitrocellulose, and stained with colloidal gold (Fig. 5, lane $b$ ). A Western blot of the same material demonstrated that five of these bands reacted with convalescent sera from volunteers who had developed $S$. flexneri 2a diarrhea (Fig. 5, lane $c$ ).

78

66

51.

45_

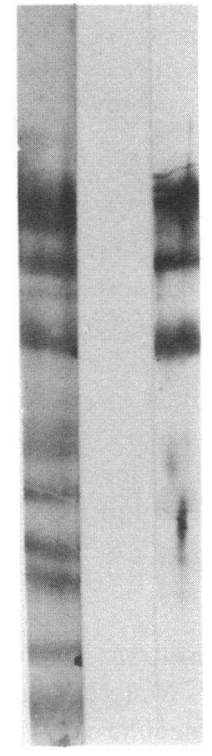

(a)

(b) (c)
Figure 5. SDS-PAGE and Western immunoblot analysis of enterotoxic fraction. The identified enterotoxic fraction from size exclusion chromatography of the $30-100-\mathrm{kD}$ batch material representing $\sim 60 \mathrm{kD}$ was separated by SDS-PAGE, and bands were transferred to nitrocellulose. Approximate molecular masses were determined using prestained markers run on the same gel (Sigma Chemical Co.) (lane $a$ ). A vertical strip was stained for total protein using colloidal gold (Aurodye; Janssen Pharmaceutica, Piscataway, NJ) (lane $b$ ). An additional strip was developed as a Western immunoblot using pooled sera from volunteers convalescing from $S$. flexneri $2 \mathrm{a}$ infection (lane $c$ ).
Cloning and sequencing the genes encoding ShET1

Identification of clones expressing ShET1 from a S. flexneri $2 a$ strain 2457TA library. A AZapII library of genomic DNA obtained from the plasmid-cured S. flexneri 2a strain 2457TA was screened by plaque immunoblot, using the rabbit polyclonal antiserum described in Methods. Of 4,000 plaques screened, 6 gave strongly positive reaction with the antibodies. Filtersterilized supernatants from six arbitrarily selected reactive clones of one of the positive plaques were tested on rabbit ileal mucosa in Ussing chambers. One of these supernatants, DH5 $\alpha$ (pF9-1-90), induced $I_{\mathrm{sc}}$ changes $\left(58.7 \pm 7.9 \mu \mathrm{Amp} / \mathrm{cm}^{2}\right)$ significantly higher than DH5 $\alpha$ negative control supernatants $\left(17.9 \pm 7.3 \mu \mathrm{Amp} / \mathrm{cm}^{2} ; P<0.01\right)$ and equivalent to $2457 \mathrm{TA}$ supernatant $\left(38.8 \pm 10.1 \mu \mathrm{Amp} / \mathrm{cm}^{2}\right)$. The $I_{\mathrm{sc}}$ changes induced by $\mathrm{DH} 5 \alpha(\mathrm{pF} 9-1-90)$ supernatant were neutralized by pretreatment with rabbit antisera (data not shown). The plasmid contained in this clone, designated $\mathrm{pF9}-1-90$, was purified and mapped, and a 6-kb DNA insert was found.

Western immunoblots of supernatants from DH5 $\alpha(\mathrm{pF} 9-1-$ 90) (Fig. $6 A$, lane $d$ ) demonstrated the expression of a $55-\mathrm{kD}$ band that was also observed in 2457TA (lane $e$ ) but not in the DH5 $\alpha$ (pBluescript SK \pm ) host strain lacking the insert (lane $a)$. A Western blot performed with pooled convalescent sera from volunteers challenged with wild-type $S$. flexneri 2 a also revealed the $55-\mathrm{kD}$ band (Fig. $6 B$, lane $b$ ). Fewer bands were recognized in the immunoblot using the human sera.

Sequencing of set. Sequencing analysis of the 6-kb insert in pF9-1-90 revealed two orfs of $186 \mathrm{bp}$ ( $\operatorname{set} 1 \mathrm{~B}$ ) and $534 \mathrm{bp}$ ( $\operatorname{set} 1 \mathrm{~A})$ in the same orientation and separated by only $6 \mathrm{bp}$ (Fig. 7). The predicted molecular masses of the protein molecules encoded by these orfs are $\sim 7$ and $20 \mathrm{kD}$ for set $1 \mathrm{~B}$ and $\operatorname{set} 1 \mathrm{~A}$, respectively. The finding of a $55-\mathrm{kD}$ protein (Fig. $6 \mathrm{~A}$ ) supports the concept of an $A_{1}-B_{5}$ configuration for the holotoxin in which the A subunit is $20 \mathrm{kD}$ and the individual $B$ subunit is $7 \mathrm{kD}$. The set $1 \mathrm{~B}$ gene has an upstream promoter governing the transcription of both set $1 \mathrm{~B}$ and set $1 \mathrm{~A}$ genes (Fig. 7). Analysis of the amino acid sequence revealed a peptide structure with a predicted signal sequence. Comparison of the predicted protein 


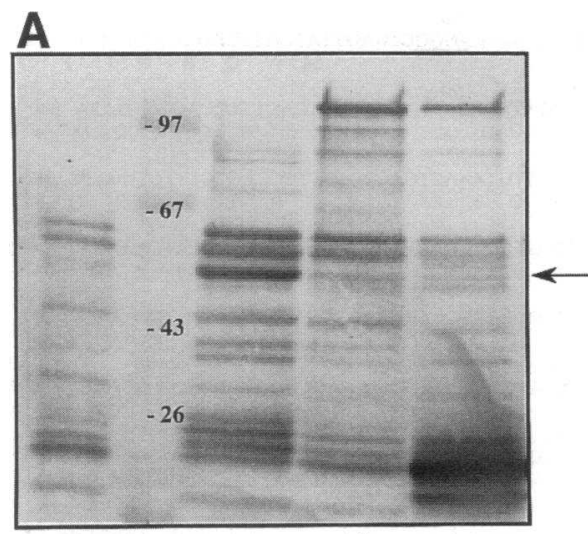

(a) (b) (c) (d) (e)

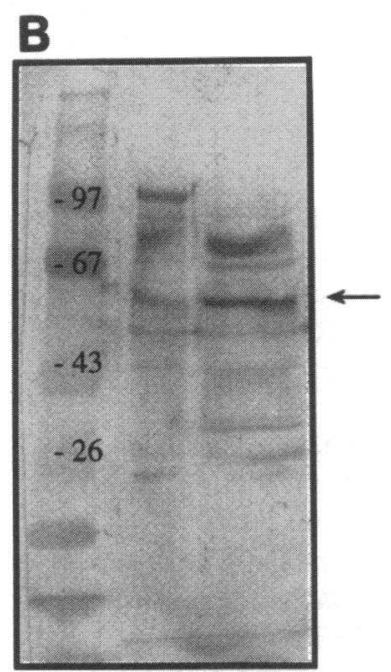

(a) (b) (c)

Figure 6. Western immunoblot of supernatants from wild-type and cloned ShET1. (A) Western immunoblot with rabbit antisera. Supernatants were separated by SDS-PAGE, transferred to nitrocellulose, and developed using rabbit antisera to the $60-\mathrm{kD}$ active fraction obtained from M4243avir. Lane $a$, pBluescript in DH5 $\alpha$; lane $b$, apparent molecular masses, prestained markers; lane $c$, pset 1 ; lane $d$, pF9-1-90; lane $e$, 2457TA. Arrow shows the $55 \mathrm{kD}$ band present in all lanes except lane $a$. (B) Western immunoblot with pooled sera from volunteers. Supernatants were separated by SDS-PAGE, transferred to nitrocellulose, and developed using pooled sera from the volunteers convalescing from $S$. flexneri 2a infection. Lane $a$, apparent molecular masses, prestained markers; lane $b, \mathrm{pF} 9-1-90$; lane $c$, pset 1 . Arrow shows 55-kD band present in lanes $b$ and $c$.

with the EMBL/GenBank library of sequences showed no significant homologies among prokaryotic or eukaryotic sequences at the amino acid or nucleotide level. The set $1 \mathrm{~A}$ gene has its own Shine-Dalgarno sequence $13 \mathrm{bp}$ upstream of the initiation codon. The predicted amino acid sequence also features a putative signal sequence. Comparison of this orf with the EMBL/ GenBank did not reveal significant homologies with known sequences.

Cloning of set genes. set orfs were cloned in pBluescript \pm , and the plasmid so obtained was transformed in DH5 $\alpha$. Immunoblots of the iron-depleted supernatant obtained from the DH5 $\alpha$ (pset 1 ) culture revealed the expression of the $55-\mathrm{kD}$ pro- tein band (Fig. $6 A$, lane $c$; Fig. $6 B$, lane $c$ ), which was also detected in $S$. flexneri 2a strain 2457TA and pF9-1-90 supernatant, but not in the DH5 $\alpha$ negative control (Fig. $6 A$, lane $a$ ).

DH5 $\alpha$ (pset 1) supernatant induced an increase in $I_{\text {sc }}$ when tested in Ussing chambers $\left(79.18 \pm 14.1 \mu \mathrm{Amp} / \mathrm{cm}^{2} ; n=6\right)$ greater than that seen with $S$. flexneri 2 a wild-type strain 2457TA (38.80 $\left.\pm 7.6 \mu \mathrm{Amp} / \mathrm{cm}^{2} ; n=6\right)$ and DH5 $\alpha(\mathrm{pF} 9-1$ 90) $\left(53.63 \pm 11.3 \mu \mathrm{Amp} / \mathrm{cm}^{2} ; n=8\right)$. All ShET1-containing supernatants tested in Ussing chambers showed a greater increase of $I_{\text {sc }}$ as compared with the changes induced by supernatants obtained from the DH5 $\alpha(\mathrm{pBS})$ negative control $\left(10.18 \pm 8.5 \mu \mathrm{Amp} / \mathrm{cm}^{2} ; n=7 ; P<0.01\right)$. Material eluted from the nitrocellulose, corresponding to the $55-\mathrm{kD}$ immunoreactive band (Fig. $6 A$, lane $c$ ), induced a significant rise in $I_{\text {sc }}$ when tested in Ussing chambers $\left(70.4 \mu \mathrm{Amp} / \mathrm{cm}^{2}\right.$ average $)$. It is interesting to note that the enterotoxic effect was proportional to the level of expression of ShET1 (pset 1 > pF9-1-90 $>$ 2457TA), suggesting a dose-response relationship for the toxicity of ShET1.

\section{Discussion}

Much has been written about the molecular pathogenesis of Shigella with respect to the genes and gene products involved in their ability to invade epithelial cells and thereby to cause dysentery (20-24). In contrast, surprisingly little is known of the precise mechanisms by which Shigella cause watery diarrhea. It has previously been hypothesized that Shigella produce an enterotoxin. However, except for the cytotoxin/neurotoxin/ enterotoxin elaborated by $S$. dysenteriae 1 (25-27), little convincing proof has been generated to substantiate the contention that these organisms in fact produce enterotoxins. Nevertheless, several observations have strongly predicted the existence of enterotoxins in $S$. flexneri:

(a) Clinically in humans, S. flexneri infections are usually characterized by a period of watery diarrhea that precedes the onset of scanty dysenteric stools of blood and mucus $(7,28)$. Indeed, in mild cases, only watery diarrhea may occur, leading to a clinical picture indistinguishable from enterotoxigenic $E$. coli infection $(29,30)$.

(b) When $S$. flexneri $2 \mathrm{a}$ are fed to monkeys, three clinical syndromes are seen (1). Some monkeys develop only dysentery, some exhibit only watery diarrhea, and some manifest both diarrhea and dysentery. In vivo perfusion studies by Rout et al. (1) showed that net transport of water into the lumen of the colon occurs in all ill animals. In contrast, only in the jejunum of monkeys with overt watery diarrhea (alone or followed by dysentery) does there occur net secretion of water, sodium, and chloride ions; such net transport does not occur in the jejunum of monkeys manifesting dysentery without watery diarrhea. Net secretion in the jejunum was not accompanied by abnormal histological findings in this anatomic site of the small intestine.

(c) The net secretion of water and electrolytes into the jejunum of monkeys with watery diarrhea requires the passage of $S$. flexneri 2a through the jejunum (2). This was elegantly demonstrated by Kinsey et al. (2), who bypassed the small intestine and inoculated monkeys directly into the cecum with $S$. flexneri 2a. Of 16 monkeys who developed clinical illness, 15 manifested dysentery, " . . . only rarely preceded by mild diarrhea." Net secretion of water and sodium into the colon was recorded in ill monkeys that developed dysentery after 
Figure 7. The complete nucleotide sequence of the $S$. flexneri $2 \mathrm{a}$ set $1 \mathrm{~B}$ and set $1 \mathrm{~A}$ genes and the corresponding deduced amino acid sequence of ShET1. Nucleotides are numbered in the $5^{\prime}$ to $3^{\prime}$ direction. Underlined sequences indicate the -35 and -10 and the first Shine-Dalgarno ( $S D$ ) regions of the single promoter upstream of set $1 \mathrm{~B}$. A second Shine-Dalgarno sequence was found 13 bp upstream of the initiation codon of set $1 \mathrm{~A}$ (not indicated). The nucleotide sequence reported will appear in the EMBL, GeneBank, and DDBJ Nucleotide Sequence databases under the accession number Z47381 authorin submission SHET.

intracecal inoculation, whereas no abnormalities of water or electrolyte transport were observed in the jejunum of the ill animals.

Together, these observations strongly suggest that Shigella elaborate enterotoxins that elicit secretion early in the infection as the organisms pass through the jejunum. Our studies validate these earlier physiologic observations, since we now demonstrate that supernatants of $S$. flexneri 2 a contain at least two distinct enterotoxins active both in Ussing chamber experiments (in which significant changes in $I_{\text {sc }}$ were recorded) and in isolated rabbit ileal loops (in which significant fluid accumulation was documented). The fluid/gut length ratios recorded in the rabbit ileal loops (average of $0.5 \mathrm{ml} / \mathrm{cm}$ ) are substantially less than those seen with enterotoxigenic $E$. coli or EHEC, in which ratios of $1.5-2.0 \mathrm{ml} / \mathrm{cm}$ occur. Nevertheless, this still represents definite, albeit mild, net secretion and fluid accumulation.

Plasmid-cured derivatives of strain M4243 showed less enterotoxic activity compared with the wild type in both ileal loops and Ussing chambers. We have recently established that the enhanced enterotoxic activity conferred upon $S$. flexneri $2 \mathrm{a}$ by its virulence plasmid reflects the presence of a plasmid gene encoding a distinct enterotoxin (ShET2 [5]).

Convalescent sera from volunteers who were fed wild-type S. flexneri $2 \mathrm{a} \cdot$ were shown to contain antibodies that neutralize the enterotoxic activity of plasmid-minus $S$. flexneri 2a supernatants in Ussing chambers. Moreover, these convalescent sera contained antibodies that specifically bind to immobilized protein shown to produce enterotoxic activity. At the least, these serologic responses confirm the expression of ShET1 in vivo in the course of human infection, even if they cannot be construed as constituting proof that the toxin actually mediates intestinal secretion. Given the plethora of virulence properties exhibited by virulent Shigella and the complexity of pathogenesis that involves interaction with the host, it will be difficult to ascribe a specific role to ShET1. Nevertheless, we plan to undertake clinical studies in the future that will attempt to assess the actual pathogenic role of ShET1. These include: (a) a randomized, double-blind volunteer study involving oral challenge of subjects with either wild-type $S$. flexneri 2 a or a mutant harboring a deletion in set and $(b)$ an intestinal perfusion study in which intestinal secretion will be measured in volunteers ran- domly allocated to be exposed to partially purified ShET1 (30$100 \mathrm{kD}$ retentate of plasmid-minus $S$. flexneri $2 \mathrm{a}$ ) or to a control preparation (an analogous retentate prepared from an isogenic mutant in which set is inactivated). Results of these planned studies may allow a pathophysiologic role to be attributed to ShET1.

Earlier attempts by other investigators to detect enterotoxic activity in supernatants of $S$. flexneri yielded negative results with the exception of reports from two groups (31-34). In none of these reports is there a resemblance to the findings reported herein for a novel putative $S$. flexneri 2 a enterotoxin for which we suggest the abbreviation ShET1. Specifically, Ketyi et al. (32-34) reported heat-stable enterotoxin elaborated by $S$. flexneri $3 \mathrm{a}$ and $2 \mathrm{a}$ strains that caused dilatation of rabbit ileal loops at $4 \mathrm{~h}$ but not at 18-24 $\mathrm{h}$ (when the effects that we observed were maximal). O'Brien et al. (31) reported a partially purified toxin prepared from $S$. flexneri 2a strain M4243 that showed cytotoxic activity against HeLa cells which was partially neutralized by Shiga toxin antiserum. This material elicited positive rabbit ileal loops (it was not stated whether these were read at $4 \mathrm{~h}$ or at 18-24 h) and was lethal for mice. The ShET1 rabbit ileal loop activity that we observed was clearly separable from any cytotoxic activity elaborated in M4243 supernatants. Moreover, neither of the earlier groups used the conditions of iron limitation that appear to be necessary for expression of ShET1 and ShET2. In summary, the few previous reports of enterotoxic activity exhibited by $S$. flexneri 2a appear to involve entities distinct from ShET1.

Analysis of the sequence of the cloned genes responsible for ShET1 enterotoxic activity revealed two distinct, yet contiguous, orfs encoding two proteins of 7 and $20 \mathrm{kD}$. Similarly, Western blot experiments (Fig. 6) are consistent with one ShET1 gene product having a size of $\sim 20 \mathrm{kD}$ and another gene product being $7 \mathrm{kD}$ in size. It is intriguing to speculate that ShET1 may exhibit the $A_{1}-B_{n}$ active binding subunit motif commonly encountered among bacterial enterotoxins, including cholera toxin (35), heat-labile enterotoxin of enterotoxigenic E. coli (36), and Shiga toxin of $S$. dysenteriae $1(37,38)$. By extrapolation, since the ShET1 holotoxin has an apparent size of $55 \mathrm{kD}$, it is tantalizing to speculate that an $A_{1}-B_{5}$ configuration of one $20-\mathrm{kD}$ subunit (typical of the size of $A$ subunits of 
other enterotoxins) bonded to five $B$ subunits, each $7 \mathrm{kD}$ in size (typical of B subunits of other enterotoxins), may be responsible. At present this must be regarded purely as speculation. However, important aims of our further research will be to characterize the physical properties of the ShET1 holotoxin and to determine the role of the gene product of each of the two orfs.

We recognize that in the current study our characterization of the properties of the enterotoxin expressed by $S$. flexneri $2 \mathrm{a}$ after growth in iron-limited medium represents a preliminary finding. More intensive investigations into ShET1 and its mode of action await development of preparative purification methods. Studies are underway to characterize further the regulation and biochemistry of this new enterotoxin.

\section{Acknowledgments}

We express our gratitude to individuals and institutions at the University of Maryland for providing necessary assistance and helpful discussions in the following areas: Edward M. Sybert and Terry Chase at the Bioprocess Scale-up Facility, Engineering Research Center, Department of Engineering, College Park, for scale-up fermentation, harvest, and ultrafiltration. We are additionally indebted to Bernardino Rea, University of Catanzaro School of Medicine, and Liao Fang-Ming, University of Maryland, for dedicated technical assistance.

This study was supported by National Institute of Allergy and Infectious Disease Salmonella typhi R01 AI29471 to M. M. Levine.

\section{References}

1. Rout, W. R., S. B. Formal, R. A. Giannella, and G. J. Dammin. 1975. Pathophysiology of Shigella diarrhea in the Rhesus monkey: intestinal transport, morphological, and bacteriological studies. Gastroenterology. 68:270-278.

2. Kinsey, M. D., S. B. Formal, G. J. Dammin, and R. A. Giannella. 1976. Fluid and electrolyte transport in Rhesus monkeys challenged intracecally with Shigella 2a. Infect. Immun. 14:368-371.

3. Fasano, A., B. A. Kay, B. G. Russell, D. R. Maneval, Jr., and M. M. Levine. 1991. Enterotoxin and cytotoxin production by enteroinvasive Escherichia coli. Infect. Immun. 58:3717-3723.

4. Levine, M. M. 1987. Escherichia coli that cause diarrhea: enterotoxigenic, enteropathogenic, enteroinvasive, enterohemorrhagic and enteroadherent. $J$. Infect. Dis. 155:377-389.

5. Nataro, J. P., J. Seriwatana, A. Fasano, F. Noriega, L. Guers, and J. G. Morris, Jr. 1993. Cloning and sequencing of a new plasmid-encoded enterotoxin in enteroinvasive E. coli and Shigella. Proc. 29th Joint Conference on Cholera and Related Diseases. 144-147. (Abstr.)

6. Andrews, G. P., A. E. Hromockyj, C. Coker, and A. T. Maurelli. 1991. Two novel virulence loci, $m x i A$ and $m x i B$, in S. flexneri $2 \mathrm{a}$ facilitate excretion of invasion plasmid antigens. Infect. Immun. 59:1997-2005.

7. Du Pont, H. L., R. B. Hornick, A. T. Dawkins, M. J. Snyder, and S. B Formal. 1969. The response of man to virulent Shigella flexneri 2a. J. Infect. Dis. 119:296-299.

8. Levine, M. M., E. J. Bergquist, D. R. Nalin, D. H. Waterman, R. B. Hornick, C. R. Young, S. Sotman, and B. Rowe. 1978. Escherichia coli strains that cause diarrhoea but do not produce heat-labile or heat-stable enterotoxins and are noninvasive. Lancet. I:1119-1122.

9. Levine, M. M., J. B. Kaper, H. Lockman, R. E. Black, and M. L. Clements 1983. Recombinant DNA risk assessment studies in man: efficacy of poorlymobilized plasmids in biologic containment. J. Infect. Dis. 148:699-709.

10. O'Brien, A. D., G. D. La Veck, M. R. Thompson, and S. B. Formal. 1982 Production of Shigella dysenteriae type 1-cytotoxin by Escherichia coli. J. Infect. Dis. 136:763-769.

11. Kotloff, K., D. Herrington, T. L. Hale, J. W. Newland, L. Van de Verg, J. P. Cogan, P. J. Snoy, J. C. Sadoff, S. B. Formal, and M. M. Levine. 1992. Safety, immunogenicity, and efficacy in monkeys and humans of invasive Escherichia coli $\mathrm{K}-12$ hybrid vaccine candidates expressing $S$. flexneri 2a somatic antigen. Infect. Immun. 60:2218-2224.

12. Guandalini, S., A. Fasano, M. Migliavacca, G. Marchesano, A. Ferola, and A. Rubino. 1987. Effects of Berberine on basal and secretagogue-modified ion transport in the rabbit ileum in vitro. J. Pediatr. Gastrenterol. Nutr. 6:953960.

13. Gentry, M. K., and J. M. Dalrymple. 1980. Quantitative microtiter cytotoxicity assay for Shigella toxin. J. Clin. Microbiol. 12:361-366.

14. Bradford, M. M. 1976. A rapid and sensitive method for the quantitation of micrograms quantities of protein utilizing the principle of protein dye binding. Anal. Biochem. 72:248-254.

15. Rogers, H. J. 1973. Iron-binding catechols in Escherichia coli. Infect. Immun. 7:445-456.

16. Laemmli, U. K. 1970. Cleavage of structural proteins during the assembly of the head of bacteriophage T4. Nature (Lond.). 227:680-685.

17. Towbin, H., T. Staehelin, and J. Gordon. 1979. Electrophoretic transfer of proteins from polyacrylamide gels to nitrocellulose sheets: procedure and some applications. Proc. Natl. Acad. Sci. USA. 76:4350-4354.

18. Vial, P. A., R. Robins-Browne, H. Lior, V. Prado, J. B. Kaper, J. P. Nataro, D. Maneval, A. Elsayed, and M. M. Levine. 1988. Characterization of enteroadherent-aggregative Escherichia coli, a putative agent of diarrheal disease. J. Infect. Dis. 158:70-79.

19. Sambrook, J., E. F. Fritsch, and T. Maniatis. 1989. Molecular Cloning. A Laboratory Manual. Second edition. Cold Spring Harbor Laboratory Press, Cold Spring Harbor, NY. 12.16-12.20.

20. Makino, S., C. Sasakawa, and M. Yoshikawa. 1988. Genetic relatedness of the basic replicon of the virulence plasmid in the shigellae and enteroinvasive Escherichia coli. Microbiol. Pathog. 5:267-274.

21. Sansonetti, P. J., D. J. Kopecko, and S. B. Formal. 1982. Involvement of a plasmid in the invasive ability of S. flexneri. Infect. Immun. 35:852-860.

22. Hale, T. L., P. J. Sansonetti, P. A. Schad, S. Austin, and S. B. Formal. 1983. Characterization of virulence plasmids and plasmid-associated outer membrane proteins in S. flexneri, Shigella sonnei and Escherichia coli. Infect. Immun. 40:340-350.

23. Pal, T., S. B. Formal, and T. L. Hale. 1989. Characterization of virulence marker antigen of Shigella spp. and enteroinvasive Escherichia coli. J. Clin. Microbiol. 27:561-563.

24. Venkatesan, M. M., J. M. Buysse, and D. J. Kopecko. 1988. Characterization of invasion plasmid antigen genes (ipaBCD) from S. flexneri. Proc. Natl. Acad. Sci. USA. 85:9317-9321.

25. O'Brien, A. D., and R. Holmes. 1987. Shiga and Shiga-like toxins. Microbiol. Rev. 51:206-220.

26. Keusch, G. T., A. Donohue-Rolfe, and M. Jacewicz. 1982. Shigella toxin(s): description and role in diarrhea and dysentery. Pharmac. Ther. 15:403438

27. Fontaine, A., J. Arondel, and P. J. Sansonetti. 1988. Role of Shiga-toxin in the pathogenesis of bacillary dysentery, studied by using a tox ${ }^{-}$mutant of Shigella dysenteriae 1. Infect. Immun. 56:3099-3109.

28. Stoll, B. J., R. I. Glass, M. I. Huq, M. U. Khan, H. Banu, and J. Holt. 1982. Epidemiological and clinical features of patients infected with Shigella who attended a diarrheal disease Hospital in Bangladesh. J. Infect. Dis. 146:177-183.

29. Taylor, D. N., P. Echeverria, T. Pal, O. Sethabutr, S. Saiborisuth, S. Srichamorn, B. Rowe, and J. Cross. 1986. The role of Shigella spp, enteroinvasive Escherichia coli and other enteropathogens as causes of childhood dysentery in Thailand. J. Infect. Dis. 153:1132-1138.

30. Taylor, D. N., P. Echeverria, O. Sethabutr, C. Pitarangsi, U. Leksomboon, N. Blacklow, B. Rowe, R. Gross, and J. Cross. 1988. Clinical and microbiological features of Shigella and enteroinvasive Escherichia coli infections detected by DNA hybridization. J. Clin. Microbiol. 26:1362-1366.

31. O'Brien, A. D., M. R. Thompson, P. Gemski, B. P. Doctor, and S. B. Formal. 1977. Biological properties of $S$. flexneri 2a toxin and its serological relationship to Shigella dysenteriae 1 toxin. Infect. Immun. 15:796-798.

32. Ketyi, I., I. Malovics, A. Vertenyi, T. Kontrohr, S. Pacsa, and B. Kuch. 1978. Heat-stable enterotoxin produced by S. flexneri. Acta Microbiol. Acad. Sci. Hung. 25:165-171.

33. Ketyi, I., A. Vertenyi, I. Malovics, T. Kontrohr, and S. Pacsa. 1978. Unique features of heat-stable enterotoxin of S. flexneri. Acta Microbiol. Acad. Sci. Hung. 25:219-227.

34. Ketyi, I., A. Vertenyi, S. Pacsa, and B. Kocsis. 1978. Enterotoxin production by S. flexneri 2 A strain no. M4243. Acta Microbiol. Acad. Sci. Hung. 25:319325 .

35. LoSpalluto, J. J., and R. A. Finkelstein. 1972. Chemical and physical properties of cholera exo-enterotoxin (choleragen) and its spontaneously formed toxoid (choleragenoid). Biochem. Biophys. Acta. 257:158-166.

36. Clements, J. D., D. C. Flint, and A. F. Klipstein. 1982. Immunological and physicochemical characterization of heat-labile enterotoxins isolated from two strains of Escherichia coli. Infect. Immun. 38:806-809.

37. Olsnes, S., R. Reisbig, and K. Eiklid. 1981. Subunit structure of Shigella cytotoxin. J. Biol. Chem. 256:8732-8738.

38. Seidah, N. G., A. Donohue-Rolfe, C. Lazure, F. Auclair, G. T. Keusch, and M. Chretein. 1986. Complete amino acid sequence of Shigella toxin-B chain. A novel polypeptide containing 69 amino acid and one disulfide bridge. J. Biol. Chem. 261:13928-13931. 